\title{
Vom Text zum Bild - Dramapädagogische Methoden im DaF-Unterricht
}

\author{
Renata Cieslak
}

\begin{abstract}
Zusammenfassung
In zahlreichen Lehrwerken für Deutsch als Fremdsprache werden Lesetexten Bilder und Bildercollagen mit dem Ziel vorangestellt, durch die vorausgehende Beschäftigung mit den Fotos das Textverständnis zu erleichtern und es durch die wiederholte Betrachtung der Fotos nach der Lektüre zu festigen. Solch eine Vorgehensweise schränkt die Imagination der Lernenden ein, die in der Didaktik als elementare Voraussetzung für das Lesen und Textverstehen anerkannt ist. Dramapädagogische Methoden können zur Förderung der Vorstellungsbildung eingesetzt werden. An einem Beispiel aus dem Lehrwerk Tangram 2B für den DaF-Unterricht soll in diesem Beitrag gezeigt werden, wie die Lernenden mithilfe der Techniken des szenischen Spiels angeregt werden können, beim Lesen des Textes eigene Bilder, Vorstellungen und Assoziationen zu entwickeln und diese sichtbar und kommunizierbar zu machen. Der Weg führt hier also vom Text zum Bild.
\end{abstract}

\section{Einleitung}

Für den Einsatz von Rollenbiographien, Gedanken-Stopps, Stimmenskulpturen, Standbildern und Statuen im Fremdsprachenunterricht sprechen viele Argumente. Der größte Vorteil dieser Übungsformen besteht in ihrer Ganzheitlichkeit. Im Klassenraum werden Interaktionssituationen geschaffen, in die sich die Lernenden mit ihren Emotionen, Vorstellungen und ihrem Weltwissen einbringen können. Sie bekommen die Möglichkeit, die fremde Sprache in Verbindung mit Emotionen und Handlungen zu erfahren. Diese Methoden fördern außerdem die affektive Seite des Lernens. Einerseits kann jeder seine eigenen Vorstellungen, Assoziationen und Emotionen in die Interpretation des Textes oder in die vorgegebene Situation einbringen. Andererseits können durch den spielerischen Charakter der Aufgaben und das Handeln in Rollen Ängste ausgeschaltet oder zumindest reduziert werden. Sprechhemmungen können überwunden werden, indem die Lernenden zum Textverständnis keine Fragen zu beantworten brauchen, sondern stattdessen ihre im ersten Rezeptionsschritt entstandenen inneren Bilder mit Hilfe von Standbildern zeigen. 
Der Einsatz dramapädagogischer Methoden eröffnet Freiräume, in denen Emotionen erlebt, gezeigt und reflektiert sowie emotionsbeladene Handlungsweisen ausprobiert und mit den Handlungsweisen anderer konfrontiert werden können. Dadurch leisten dramapädagogische Methoden einen erheblichen Beitrag zum Erwerb von Kompetenzen, welche die Fremdsprachenlernenden in die Lage versetzen, in kommunikativen Situationen angemessen zu agieren und Kommunikationsprobleme zu bewältigen. Aspekte wie interpersonale und interkulturelle Beziehungen, Körpersprache oder rituelles Verhalten können so erlebbar und erfahrbar gemacht werden. Ausgewählte kulturspezifische Aspekte der Kommunikation können in einzelnen Handlungsweisen umgesetzt und in gespielten Begegnungssituationen und einer anschließende Reflexionsphase ins Bewusstsein der Lernenden gebracht werden. Die Verbindung von Sprachhandlungen und motorischer Aktivität macht soziale oder kulturelle Unterschiede besser erfahrbar als eine ausschließlich kognitive Vorgehensweise. Sind sich die Lernenden der Unterschiede bewusst, ist davon auszugehen, dass sie entsprechende Sprachregister sicherer anwenden können, um sich situationsangemessen auszudrücken. ${ }^{1}$

\section{Dramapädagogik und Vorstellungsbildung}

Über das bereits geschilderte Potenzial von dramapädagogischen Methoden im Fremdsprachenunterricht hinaus, kommt diesen im Hinblick auf die Vorstellungsbildung als einer wichtigen Voraussetzung für die Bildung und Entwicklung des Menschen eine große Bedeutung zu. Nach Kants Auffassung spielt die Vorstellungskraft für unsere Wahrnehmung und unser Denken eine zentrale Rolle: Ohne sie könnten wir weder etwas wahrnehmen noch etwas denken, sie sei die Grundlage mentaler Abläufe. Auch in der Pädagogik und der Literaturdidaktik liegen didaktische Konzepte vor, deren Ziel es u.a. ist, die Vorstellungskraft der Lernenden zu fördern (cf. Madelung 1996: 109; Spinner 1997). Die Literaturdidaktik formulierte ihre Postulate nach der Entwicklung der Fähigkeit zur Imagination (cf. Spinner 1993, 1997) bzw. zur Vorstellungsbildung (cf. Abraham 1999) im Anschluss an die rezeptionsästhetische Folgerung, dass Leerstellen in literarischen Texten erst von den RezipientInnen in der Fantasie gefüllt werden. Folglich wird der Literaturunterricht auch als ein Ort der Vorstellungsbildung und Vorstellungsschulung gesehen. Die Aufwertung der Rolle des Lesers bzw. der Leserin bei der Bedeutungskonstitution des Textes hatte ebenfalls zur Folge, dass man in der Literaturdidaktik heute den Bildern, die während der Lektüre eines Textes im Kopf des Lesers bzw. der Leserin entstehen, einen höheren Stellenwert zuschreibt als den Bildern, Fotos oder Abbildungen in einem Lehrwerk. ${ }^{2}$

\footnotetext{
1 Zum Potenzial dramapädagogischer Methoden im Fremdsprachenunterricht siehe auch Schewe 1993: 412-423.

2 Die Aufwertung der inneren gegenüber den realen Bildern in der Literaturdidaktik geht auf Iser (1984: 223) zurück, der das innere Bild von der optischen Wahrnehmung abgrenzte und ihm als der zentralen Kategorie der Vorstellung größere Bedeutung beigemessen hat.
} 


\section{Bildmaterial im Fremdsprachenunterricht}

In den Lehrwerken für Deutsch als Fremdsprache spielen Fotos und Bildercollagen grundsätzlich zwei Rollen: Sie werden ganzen Kapiteln als Aufmacher vorangestellt oder begleiten Lesetexte. Sie führen also in die neue Thematik ein, ermöglichen es, Assoziationen zu sammeln und auszutauschen sowie den neuen Wortschatz zu vermitteln. Sie erleichtern das Verstehen des gelesenen Textes, indem sie das im Text beschriebene Geschehen illustrieren. Die Arbeit mit dem Bildmaterial erfolgt meistens mithilfe verschiedener Brainstorming-Verfahren wie Mind-Mapping oder Clustering. Vor dem Lesen des Textes werden die Lernenden angeregt, anhand des Bildes Vermutungen über die Figuren oder den Verlauf der im Text erzählten Geschichte zu formulieren. Nach der Lektüre wird gewöhnlich überprüft, inwieweit die früheren Vermutungen zutreffen. $\mathrm{Zu}$ den üblichen Übungen gehört auch die Zuordnung von Überschriften oder Textfragmenten zu den Bildern. Der Einsatz dieser populären pre- und postreading-Aufgaben bei der Textarbeit im Fremdsprachenunterricht kann dazu führen, dass diejenigen Lernenden, die vor der Lektüre eines Textes seinen Inhalt in Bildform rezipiert haben, kaum noch angeregt werden, eigene Fantasien zu dem im Text Dargestellten zu entfalten. Dramapädagogische Verfahren können hingegen helfen, in einem Fremdsprachenunterricht, in dem besonders viel mit Bild- und Textmaterial gearbeitet wird, die Imaginationsfähigkeiten der Lernenden zu fördern. In dramapädagogischen Ansätzen stellen die Aufgaben zur Vorstellungsbildung einen unabdingbaren, der eigentlichen Analyse des Textes vorausgehenden Teil der Interpretationsarbeit dar. Dies resultiert aus der Überzeugung, dass die „sinnliche Vorstellung des Textes“ und „die Entwicklung von räumlich-szenischer Imagination“ (Kunz 1995: 57) eine Voraussetzung für die Interpretation bilden.

\section{Unterrichtspraktische Beispiele}

Im Folgenden möchte ich am Beispiel der Lektion 9 „Konflikte und Lösungen“ aus dem Lehrwerk Tangram 2B Möglichkeiten für den Einsatz dramapädagogischer Methoden im DaF-Unterricht aufzeigen. Die einzelnen Techniken habe ich Ingo Schellers (2004) Konzept der szenischen Interpretation entnommen. Schellers Methode ist einerseits in Theorie und Praxis des handlungs- und erfahrungsbezogenen Literaturunterrichts, andererseits in der Dramapädagogik verankert. Sie zielt darauf, den Lernenden einen neuen Zugang zu Texten zu eröffnen und die Texte in Anknüpfung an die persönlichen Erfahrungen und Vorstellungen zu deuten, was sie für die Fremdsprachendidaktik besonders interessant und im Fremdsprachenunterricht praktikabel macht. Das Lehrwerk Tangram $2 B$ ist auf das Sprachniveau B1 des Gemeinsamen europäischen Referenzrahmens ausgerichtet. Ich verwende dieses Lehrwerk seit fünf Jahren in meiner Tätigkeit als Deutschlehrerin, um die TeilnehmerInnen der Deutschkurse auf die Prüfung 
,Zertifikat Deutsch ${ }^{3}$ vorzubereiten. Die im Folgenden dargestellten Vorschläge zum Einsatz der Techniken des szenischen Spiels im DaF-Unterricht wurden von mir in verschiedenen Variationen sowohl mit Deutsch lernenden Jugendlichen im Alter zwischen 16 und 19 Jahren als auch mit GermanistikstudentInnen, die künftig als Deutschlehrer arbeiten möchten, bereits mehrmals erprobt.

\section{1 „Beziehungskisten“ - Tangram 2B, Arbeitsbuch, S. 115: Aufgaben der Lehrwerkautoren und Alternativvorschläge unter Einsatz dramapädagogischer Methoden}

Die Autoren des Lehrwerks schlagen vor, mit der Übung A1 im Arbeitsbuch in das Thema „Konflikte und Beziehungen“ einzusteigen. In dieser Übung findet man zehn verschiedene Zeichnungen, die jeweils ein anderes Problem in einer Partnerschaft darstellen. Im ersten Schritt sollen die SchülerInnen die Probleme benennen (z.B. 2 Untreue bzw. Eifersucht, 6 Erziehungsprobleme, 8 Streitigkeiten um die Pflichtenteilung im Haushalt) und die Gründe für die Konflikte erläutern (z.B. 1 unpassende Kleidung, 4 der Partner verspätet sich, 7 die Unfähigkeit, mit Geld umzugehen). Anschließend sollen sich die Lernenden Notizen machen und sich somit auf den zweiten Schritt vorbereiten: das Schreiben von Dialogen zu den vorgegebenen Situationen. In den drei Kästen unterhalb der Bilder finden sie Wortmaterial, das hilft, die Gespräche einzuleiten, Ärger und Unzufriedenheit auszudrücken sowie darauf zu reagieren. Die angegebenen Sätze können und sollen entweder wortwörtlich oder nach eventuell notwendigen Ergänzungen in die Dialoge eingebaut werden. Hier kann es vorkommen, dass manche Lernenden sich in ihren Dialogen auf drei Sätze - Frage/Antwort/Reaktion - beschränken. Dies ist zwar nicht negativ zu beurteilen, damit wäre aber das Potential der Zeichnungen als Sprechanlass nicht ganz ausgeschöpft.

Mithilfe dramapädagogischer Techniken kann man die Lernenden zu weiteren Sprechhandlungen anregen. Darüber hinaus möchte ich auf einen weiteren Aspekt aufmerksam machen: Das Schreiben von Dialogen bedeutet nicht, dass sich die SchülerInnen dadurch in eine authentische Kommunikationssituation versetzen können. Im herkömmlichen Unterricht werden die aufgeschriebenen Dialoge meistens nur vorgelesen. Sprechen und Handeln gehen also weit auseinander. Dies wirkt demotivierend, weil die Relevanz der Dialoge für den Einsatz in authentischen Kommunikationssituationen nicht sicht- bzw. erfahrbar wird. Dramapädagogische Techniken öffnen neue Möglichkeiten für einen wirklichkeitsnahen Sprachgebrauch, indem sie SchülerInnen dazu ermuntern, in den vorgegebenen Situationen einen Selbstbezug aufzubauen, d.h. das im Text Dargestellte mit der eigenen Erfahrungswelt zu vergleichen sowie eigene Emotionen und Erfahrungen in die Szenen einzubringen.

Damit die Lernenden sich besser in die Konfliktsituationen und die innere Welt der gezeichneten Figuren hineinversetzen können, bietet es sich an, Selbstdarstellungen oder Rollenbiographien aus der Sicht der Figuren und

\footnotetext{
${ }^{3}$,Zertifikat Deutsch` ist der offizielle Name für die B1-Prüfung des Goethe-Instituts.
} 
in der Ich-Form schreiben zu lassen. Der Schreibprozess kann durch Fragen zur Einfühlung in die Figuren gesteuert werden. Diese von der Lehrperson vorgegebenen Fragen lenken die Aufmerksamkeit auf die Gründe für den jeweiligen Konflikt und sind für das Verständnis der Figuren, ihrer Denkund Handlungsweisen grundlegend. Die Fragen sind so formuliert, dass sie zunächst das Äußere, dann die innere Welt der Figuren zu erfassen erlauben. Diese produktorientierte Aufgabe wurzelt im kreativen Schreiben. Sie aktiviert die Einbildungskraft der Lernenden, die es ihnen ermöglicht, die vorhandenen Leerstellen - Alter, Wohnort, Freizeitaktivitäten der Figuren, Aussehen und Charaktereigenschaften von deren Partner bzw. Partnerin, Erfahrungen und Erwartungen in Bezug auf den Partner bzw. die Partnerin - fantasievoll zu füllen und somit zugleich einen Selbstbezug herzustellen. Dies erfolgt zwangsläufig, weil die Lernenden beim Schreiben der Rollenbiographien auf ihr Weltwissen, ihre Erfahrungen im Umgang mit anderen Menschen und auf ihnen bekannte Konfliktlösungsstrategien zurückgreifen und sie in ihre Darstellungen einbeziehen.

Im Anschluss an die Rollenbiographien kann noch die individuelle Sprechweise einer Figur im Hinblick auf deren Lautstärke und Intonation erarbeitet werden. Das Wortmaterial in den drei Kästen eignet sich dafür sehr gut. Die einzelnen Sätze können in Verbindung mit einer entsprechenden Körperhaltung erprobt werden, denn, so Scheller, „körperliche Anteile der Sprache entscheiden nicht selten darüber, wie sprachliche Äußerungen gemeint sind und verstanden werden" (Scheller 2004: 113). Dieser Übung kommt aus zweierlei Gründen eine wichtige Bedeutung zu. Erstens ist es in einer Fremdsprache besonders schwierig, Emotionen und insbesondere negative Emotionen, zur Sprache zu bringen. Zweitens konnte ich in meinem Unterricht öfters beobachten, dass Fremdsprachenlernende kaum darauf achten, dem Inhalt ihrer Aussage mit ihrer Sprechweise und Körperhaltung zu mehr Expressivität zu verhelfen und sich somit verständlicher zu machen. Ein Grund hierfür mag darin liegen, dass der affektiven und interaktiven Verstehenskompetenz im Fremdsprachenunterricht zu wenig Beachtung geschenkt wird. Folglich bleiben die Lernenden meistens in der Schulbank sitzen. Aufgefordert, ihre Dialoge zu präsentieren, lesen sie die vorher vorbereiteten Texte emotionslos vor. Daher schlage ich vor, dass die Lernenden verschiedene Sprechweisen am Beispiel eines Satzes aus dem Wortschatzkasten probieren, indem sie ihn leise, laut oder schreiend, wütend oder resigniert aussprechen. Sobald sie ihren Satz und ihre individuelle Sprechweise gefunden haben, überprüfen sie, wie ihre Aussage auf andere Menschen wirkt und welche Reaktionen sie bei anderen auslösen kann. Dafür bewegen sich die Lernenden frei im Raum und sprechen ihren Satz aus, wenn sie einer anderen Person begegnen. Die angesprochene Person reagiert gemäß der Situation z.B. mit dem Satz, den sie vorher eingeübt hat.

Bevor die Dialoge szenisch umgesetzt werden, sollte zweierlei passieren: Die Figuren stellen sich vor und die Lehrperson führt mit den Lernenden Einfühlungsgespräche, die ihnen helfen, sich in die momentane Situation und Befindlichkeit ihrer Figuren einzufühlen. Die Lernenden nehmen eine auf den 
Zeichnungen vorgegebene Haltung ein, die Lehrperson beginnt ein Gespräch und tastet sich fragend daran heran, was die Figur gerade beschäftigt (cf. ibid. 69): Was tust du gerade? Was denkst du in diesem Moment? Wie fühlst du dich gerade? Was würdest du jetzt am liebsten tun? Danach kann der Dialog bzw. die Szene gespielt werden. Diese Übung geht über die Aufgabenstellung im Buch hinaus und verfolgt das Ziel, sich in die auf den Bildern vorgegebene Situation einzufühlen sowie die Unterschiede in den Erwartungen an den Partner/die Partnerin sichtbar zu machen.

Das Spiel kann außerdem mit Gedanken-Stopps unterbrochen werden. Die Lehrperson oder die Lernenden, die das Spiel beobachten, können es unterbrechen, indem sie „Stopp!“ rufen. Dann erstarren die SpielerInnen in ihren Haltungen und sprechen aus, was ihre Figur gerade denkt. Die BeobachterInnen können das Spiel auch dann unterbrechen, wenn sie den Eindruck bekommen, dass das Handeln bzw. Sprechen und Denken der Figur auseinandergehen. Sie haben die Möglichkeit, Fragen zu stellen, die ihnen zum Verständnis der Handlungsmotive der Figur verhelfen. Die SpielerInnen beantworten die Fragen spontan aus ihrer Rolle heraus. Scheller macht darauf aufmerksam, dass die Lernenden „dabei auf eigene Erlebnisse und Fantasien zurückgreifen und in der Rolle zu denken und zu empfinden beginnen“ (ibid. 70). Den BeobachterInnen hingegen gewährt diese Technik „kurze Einblicke in die Innenwelt der Figuren" (ibid. 70). Da die Lernenden hier in Rollen agieren, können sie ihre eigenen Erfahrungen und Gefühle konkretisieren, ohne als sie selbst im Mittelpunkt zu stehen. Der größte Vorteil der Technik des Gedanken-Stopps liegt meiner Meinung nach allerdings darin, dass die BeobachterInnen nicht passiv im Hintergrund verharren, sondern sich aktiv am Spiel beteiligen und somit ihre Perspektiven und Erfahrungen in das Spiel einbringen können. Dabei ergibt sich die Möglichkeit auszuprobieren, wie man negative Emotionen wie Ärger und Unzufriedenheit in einer fremden Sprache zum Ausdruck bringen und wie man mit ihnen umgehen kann.

Die vorgeschlagene Aufgabenstellung provoziert die Lernenden zu einer scharfen Auseinandersetzung mit ihrem Spiel-/Gesprächspartner. Möglicherweise befinden sich viele Lernende zum ersten Mal in einer Konfliktsituation, in der sie verbal in einer Fremdsprache agieren müssen. In einem Erlebnisgespräch zwischen dem Spielleiter und den SpielerInnen sollte daher direkt nach Beendigung einer Szene über das Erlebte reflektiert werden. Dies geschieht, indem die Figuren berichten, „wie sie das gerade vergangene Handlungsgeschehen empfunden haben, was das Erlebte für sie bedeutet und welche Handlungskonsequenzen sie daraus ziehen wollen" (ibid. 71).

\section{2 „Wir reden gerade so nett“4 - Tangram 2B, Kursbuch, S. 97-98}

Vorschläge der Lehrbuchautoren zur Textarbeit und unterstützende Maßnahmen aus dramapädagogischer Sicht — Der Einstieg in das Thema „Beziehungskonflikte“

\footnotetext{
${ }^{4}$ Der bearbeitete Text „Wir reden gerade so nett“ von Doris Dörrie bildet im Kursbuch die Grundlage der Arbeit zum Thema „Konflikte und Lösungen“.
} 
erfolgt im Kursbuch durch eine Bildercollage. Die Lernenden werden angeregt, die Fotos auf S. $97 \mathrm{zu}$ betrachten und sich mithilfe von W-Fragen einen ersten Eindruck von der Situation zu verschaffen. Als eine Variante dazu wird im Buch empfohlen, nur das erste Foto auf dem OHP zu zeigen. Die Lernenden sollen dann die Situation und die Stimmung zwischen den beiden Personen beschreiben sowie Vermutungen darüber sammeln, was vorher zwischen dem Mann und der Frau passiert ist und wie sich die Situation wohl weiter entwickeln wird. Danach werden die Lernenden dazu ermuntert, eine zu den Fotos passende Geschichte zu erfinden bzw. in Vierer-Gruppen Dialoge zu den Fotos zu schreiben. Dabei sollen sie sich für eine Rolle entscheiden, sich Notizen anfertigen und den Dialog im Plenum vorspielen.

Zusätzlich verweisen die Autoren des Lehrwerks auf eine interessante Variante des Rollenspiels, die sie „Rollenspiel mit lauten Gedanken“ nennen. Sie schlagen vor, jeder Figur, die dargestellt werden soll, nicht nur einen Schauspieler bzw. einen Sprecher, sondern auch einen Denker zuzuweisen. Eine Person solle das spielen, was ihre Figur wirklich in der Situation sagt, die Aufgabe des Denkers sei es dagegen, das laut zu sagen, was die Figur eigentlich denkt. Diese Methode ist der Psychotherapie bzw. dem Psychodrama entlehnt und hat seit Jahren einen festen Platz in der Didaktik. In ihren Stundenblättern zum Thema Rollenspiele im Literaturunterricht sprechen Dorothea Freudenreich und Fritz Sperth über die Methode des Doppelns: Sie ermögliche das, was die Wirklichkeit nicht zulasse, nämlich die Darstellung einer zweiten Ebene parallel zur Darstellung einer äußeren Handlung. In ihrem Konzept des Rollenspiels sei das Doppeln eine Hilfe für den Spieler, manchmal seine innere Stimme. Der Spieler könne sich gegen das Doppel wehren, wenn er den Eindruck gewinne, dass die beschriebenen Gefühle falsch seien (Freudenreich/Sperth 1983: 40f.).

Die gleiche Rolle, die bei den Tangram-Autoren der Denker und bei Freudenreich/Sperth das Doppel spielt, spielt im dramapädagogischen Ansatz nach Ingo Scheller das Hilfs-Ich. Scheller definiert das Hilfs-Ich als Teil und nicht inneren Partner der Figur und schreibt ihm die Aufgabe zu, Gedanken, Gefühle und Wünsche der Figur, die diese sich nicht zugesteht oder nicht auszuagieren wagt, öffentlich auszusprechen. Das Hilfs-Ich stellt sich hinter den Spieler, legt ihm die Hand auf die Schulter und spricht die Gedanken und Gefühle in der Ich-Form aus. „Spürt es, dass der Spieler die artikulierten Gefühle annehmen kann, kann es diese durch Wiederholungen und einen dringlicheren Tonfall so verstärken und unterstützen, dass der Spieler in der Rolle wagt, sie auszuagieren." (Scheller 1998: 54-55)

Im Hinblick auf den Fremdsprachenunterricht und insbesondere diese konkrete Lehrbuchaufgabe bezweifle ich, ob Lernende tatsächlich imstande sind, als Hilfs-Ich die Handlungsweise der Figuren so zu beeinflussen, wie sich das Scheller für den muttersprachlichen Deutschunterricht vorstellt. Ich vermute, dass dies auf dem Sprachniveau B1 eher problematisch ist. Eine Lösung sehe ich darin, dass die Lehrperson die Rolle des Hilfs-Ichs übernimmt. Oder aber die Lernenden spielen zwar das Hilfs-Ich, es wird aber nicht darauf bestanden, dass das jeweilige Hilfs-Ich sich um jeden Preis bemüht, den Spieler 
bzw. die Spielerin zur Veränderung seiner bzw. ihrer Handlungsweise zu bewegen. Mit der Technik des Hilfs-Ichs bzw. des Doppelns soll doch vor allem ein Zugang zur inneren Welt der Figur eröffnet werden, so dass auch blockierte Gefühle wie z.B. Ärger und Wut ausagiert werden können. Die von mir im Unterricht gesammelten Erfahrungen zeigen, dass man dieses Ziel durchaus erreichen kann, wenn man die Lernenden dazu animiert, das Alter Ego der gespielten Figur sprechen zu lassen. Beim Spielen der Dialoge bzw. Szenen hat es sich bewährt, den Sprechanteil des Alter Egos zugewandt zum Publikum bzw. zur Gruppe und den Sprechanteil der Figur dem Gesprächspartner zugewandt zu sprechen. ${ }^{5}$

Alternativvorschlag unter Einsatz dramapädagogischer Methoden -Nach der Auseinandersetzung mit der Bildercollage sollen die Lernenden gemäß der Aufgabe im Lehrbuch den Text „Wir reden gerade so nett"von Doris Dörrie auf S. 98 lesen. Der Text ist in Abschnitte eingeteilt, die jeweils von einem anderen Erzähler erzählt werden. Der erste Abschnitt soll gemeinsam gelesen werden, und die Lernenden sollen im Text Wörter unterstreichen, die darauf hindeuten, dass es sich hier um die Perspektive des Mannes handelt. Danach lesen die Lernenden den ganzen Text und weisen den Abschnitten den jeweiligen Erzähler zu. Nach der Lektüre werden die Ergebnisse im Plenum verglichen, und es wird überprüft, inwieweit die in der Übung A1 (Bildercollage) gesammelten Vermutungen zutreffen. Zum Schluss werden die Textabschnitte den Fotos zugeordnet. Da die Bildercollage und der Text jeweils auf ihre Weise dieselbe Geschichte erzählen, wird das Textverständnis durch die vorausgehende Beschäftigung mit den Fotos erleichtert und durch die wiederholte Betrachtung der Fotos nach der Lektüre gefestigt.

Ein von mir erprobter Alternativvorschlag besteht darin, den Lernenden die Bildercollage zunächst nicht zu zeigen, sondern stattdessen zu versuchen, sie mithilfe der Techniken des szenischen Spiels dazu anzuregen, beim Lesen des Textes Bilder, Vorstellungen und Assoziationen zu entwickeln und diese darzustellen. Dies entspricht der Überzeugung Schellers, dass literarische Texte nur verstanden werden können, wenn sie in der Vorstellung der LeserInnen inszeniert, d.h. in sinnlich-konkrete Bilder und Szenen verwandelt werden. Die szenische Interpretation aktiviere das sinnliche, emotionale, körperliche und szenische Gedächtnis, rege Vorstellungen über den Handlungsort und die inneren und äußeren Handlungen, Haltungen und Beziehungen der Figuren an und ermögliche dadurch die Darstellung dieser inneren Bilder (cf. Scheller 1995: 59). Allerdings weist Scheller auf ein Problem hin, das dabei entsteht. Es ist nämlich so, dass „die Inszenierungen im Kopf aufgrund des dabei verwendeten emotional besetzten Erfahrungs- und Fantasiematerials [in der Regel unscharf bleiben], Projektions- und Abwehrprozesse nicht ausschließen und sprachlich nur schwer zu vermitteln sind" (ibid.).

\footnotetext{
${ }^{5}$ Mit dieser Vorgehensweise orientiere ich mich an Bertolt Brecht, insbesondere an seiner Figur des Wasserverkäufers in Der gute Mensch von Sezuan.
} 
In der Praxis hat es sich bewährt, den Unterricht nach folgendem Szenario zu gestalten: Der Text wird von zwei Personen, die zu Hause das Vorlesen eingeübt haben, entsprechend den zwei Erzählern vorgelesen, und die anderen Lernenden werden aufgefordert, die Augen zu schließen und den Text in ihrer Vorstellungskraft in innere Bilder umzusetzen. Die Formulierung „Schließen Sie die Augen und lassen Sie einen Film in Ihrem Kopf laufen" erwies sich dabei als zielführende Aufgabenstellung.

Die Aktivierung innerer Bilder intensiviert die Begegnung mit dem Text. Dabei ist jeder gefordert, den Text für sich zu imaginieren und dann seine Vorstellungen sichtbar zu machen, indem er ein Standbild aufbaut. Standbilder kann man als eingefrorene Filmbilder bezeichnen. Die Lernenden, auch solche, die in üblichen Unterrichtsgesprächen eher schweigen, bekommen hier die Möglichkeit, Situationen, die ihnen nach der Lektüre im Kopf geblieben sind, $\mathrm{zu}$ zeigen. Jeder baut ein Standbild auf, indem er als eine Art Bildhauer seine MitschülerInnen formt, Mimik wird vorgezeigt und nachgeahmt. In den Standbildern zeigen die Lernenden das, was sie beim Zuhören verstanden und im Gedächtnis behalten haben. Öfters kommt es dabei vor, dass sich manche Textstellen gut, andere hingegen kaum eingeprägt haben. Sollte Letzteres der Fall sein, kann der Spielleiter bzw. die Lehrperson ein Standbild zu einer Textstelle aufbauen, die in der Vorstellung der Lernenden nicht in Bilder umgesetzt wurde. Ich habe in der Praxis häufig erlebt, dass das von mir aufgebaute Standbild bei den Lernenden weitere Assoziationen hervorgerufen hat, so dass schließlich ausreichend Standbilder zum Verständnis des Textes aufgebaut werden konnten. Die aufgebauten Standbilder erfüllen für das Leseverständnis des Textes eine ähnliche Funktion wie die Bildercollage auf der S. 97. Da die Lernenden aufgefordert werden, bei jedem Standbild zu raten, auf welche Situation im Text es sich bezieht, kann man diese Übung mit der herkömmlichen Aufgabenstellung vergleichen, die sich auf das Formulieren von Vermutungen zu den Bildern konzentriert. Bei der Deutung der Standbilder werden ebenfalls die Beziehungsprobleme angesprochen, und es wird die Zahl der Figuren im Text geklärt. Die Klärung des Wortschatzes findet erst vor dem zweiten Lesevorgang statt.

Bevor die Lernenden den Text zum zweiten Mal lesen (diesmal liest jeder für sich), entscheiden sie sich für eine der vier Rollen und schreiben dann - gestützt auf den Text und die Einfühlungsfragen - eine Rollenbiographie. Die Rollen können doppelt oder sogar dreifach besetzt werden, die Selbstdarstellungen werden jedoch in Einzelarbeit angefertigt. Das Schreiben von Selbstdarstellungen ermöglicht den Lernenden, sich in die Umstände, den Alltag, aber auch die Emotionen der Figuren in der jeweiligen Situation im Text einzufühlen. Dabei verhelfen die Einfühlungsfragen dazu, die Gründe für die Konflikte in den beiden Beziehungen zu erörtern. Die im Text enthaltenen Informationen über die einzelnen Figuren und ihre Probleme werden verarbeitet, und die Unbestimmtheitsstellen werden fantasievoll konkretisiert. Das Schreiben von Rollenbiographien erhöht die Identifikation mit der Figur im Text, deren Perspektive eingenommen wird (cf. Scheller 2004: 61f.), und erleichtert somit 
das spätere Handeln in der Rolle. Da das Schreiben von Rollenbiographien einen relativ großen Zeitaufwand erfordert, sollen sich die Einfühlungsfragen vorwiegend auf die im Text enthaltenen Informationen und die möglichen Konfliktgründe beziehen. Dadurch können sich die Lernenden intensiver mit dem Text auseinandersetzen und effektiver auf das Handeln in den Rollen vorbereiten.

Die Lernenden präsentieren ihre Selbstdarstellungen; zunächst das Paar 1, dann das Paar 2. Im nächsten Schritt werden die Lernenden ermuntert, sich vorzustellen, ihre Figuren befänden sich in einem Restaurant. Der Spielleiter, dessen Rolle (zunächst) von der Lehrperson übernommen wird, führt mit ihnen Einfühlungsgespräche, um ihre momentane Gefühlslage auszuarbeiten - z.B. anhand der folgenden Fragen: Wo befindest du dich gerade? Wie gefällt es dir hier? Wie fühlst du dich gerade? Welche Erwartungen hast du an den Abend? Danach wird der erste Textabschnitt laut vorgelesen. Die Lernenden stellen die Konfliktsituation zwischen dem Mann 1 und der Frau 1 mithilfe einer Statue dar. Statuen sind zwar mit den situationsbezogenen Standbildern verwandt, unterscheiden sich von diesen jedoch dadurch, dass sie von konkreten Szenen abstrahieren und nicht aus einer bestimmten Perspektive, sondern von allen Seiten wahrgenommen und gedeutet werden. Scheller bezeichnet sie als „Skulpturen, Denkmäler“ (Scheller 1998: 68) und sagt: „Sie machen abstrakte Zusammenhänge, wie Beziehungskonstellationen, generelle Haltungen der Figuren oder den Grundgestus von Szenen sichtbar und bringen sie bildlich auf den Punkt.“ (Scheller 2004: 63) Statuen werden ähnlich wie Standbilder gebaut, „wobei allerdings den Körperhaltungen, der Gestik und Mimik sowie der Personenkonstellation im Raum symbolische Bedeutung zukommt" (ibid. 73). Da Standbilder und Statuen auf dieselbe Art und Weise aufgebaut werden, erweist sich die Differenzierung zwischen den beiden Begriffen oft als problematisch. Für den Unterricht bietet es sich an, die Aufgabenstellung entsprechend der Funktion der beiden Techniken zu formulieren, z.B. Was passiert hier? Bauen Sie ein Standbild auf. Oder: In welcher Beziehung stehen die Figuren zueinander? Bauen Sie eine Statue auf.

Da es im nächsten Schritt darum gehen soll, die Gründe für die Konflikte zwischen den Figuren des Textes zu erörtern, werden die Lernenden aufgefordert, Statuen und nicht Standbilder aufzubauen. Zuerst baut also ein Schüler die Statue auf und veranschaulicht auf diese Weise, wie er die Probleme bei diesem Paar verstanden hat und worin der Grund für den Konflikt liegt. Anders als beim Stanbild erklärt er der ganzen Gruppe, wer wer ist, warum die Figuren nah oder weit voneinander stehen, sich anschauen oder nicht. Dann haben auch die anderen Lernenden die Möglichkeit, diese Statue nach ihrer Vorstellung umzugestalten, vor allem wenn sie mit der zunächst präsentierten Auffassung nicht einverstanden sind. Dadurch werden immer neue Aspekte bzw. Deutungen ins Spiel gebracht und zur Diskussion gestellt. Die Statue ist erst dann fertig, wenn die dargestellte Figurenkonstellation den Vorstellungen der meisten Lernenden nahe kommt. Diese Technik erlaubt den Lernenden also, ihre individuellen Interpretationsvorschläge zu veranschaulichen, sich mit den 
Deutungen der anderen auseinanderzusetzen und dabei Undeutlichkeiten oder Irritationen zu klären. Während die Lernenden also mit Standbildern zeigen, was sie verstanden haben, bringen sie mit Statuen ihren Interpretationsansatz zum Ausdruck.

Für eine nächste Aufgabe wird die Technik der, Stimmenskulptur' eingesetzt. In der Vorbereitungsphase lesen diejenigen Lernenden, welche die Rolle der Frau 1 übernommen haben, den Textabschnitt 2, und diejenigen, welche die Rolle des Mannes 1 übernommen haben, den Textabschnitt 3. In Gruppen wird die innere Haltung der Figuren erarbeitet: Die Lernenden überlegen, was der Frau und dem Mann in dieser Situation durch den Kopf geht, und formulieren diese Gedanken. Dabei können Sätze aus dem Text umformuliert oder aber wortwörtlich übernommen werden. Die Frau 1 und der Mann 1 setzen sich dann einander gegenüber und nehmen die Körperhaltung entsprechend der Textstellen ein (Sie: „Ich sitze auf der Klobrille und heule wie ein Schlosshund“; er: „Wie ein Idiot sitze ich da und male Muster mit der Gabel ins Tischtuch“). Die übrigen Lernenden stellen sich hinter ihre Figuren und sagen einen Satz, welcher der Figur durch den Kopf gegangen sein könnte. Wenn genug Stimmen vorhanden sind, ruft der Spielleiter diese nacheinander ab. Er dirigiert die Stimmen, indem er auf die entsprechenden RepräsentantInnen zeigt, die dann ihren Satz sagen. Die Frau 1 bzw. der Mann 1 konzentrieren sich auf die Stimmen und entscheiden, welche ihren Vorstellungen entsprechen, welche ungeeignet sind, welche entfernt, welche dagegen näher herangeholt werden sollen. Der Stimmenchor wird so lange geprobt, bis er den Vorstellungen der Frau/des Mannes entspricht (cf. Scheller 1998: 136). Der Spielleiter koordiniert die Stimmen, um sie zueinander in Beziehung zu setzen. So findet über den Köpfen des Mannes und der Frau ein Metagespräch statt, in dem die Widersprüche in den Erwartungen an den gemeinsamen Abend und in der Stimmungslage der beiden verdeutlicht werden.

Danach wird der Text zu Ende gelesen. Die Lernenden zeigen mit einer Statue die Konfliktsituation zwischen der Frau 2 und dem Mann 2. Aufbau und Deutung der Statue erfolgen nach denselben Regeln wie im Falle von dem ersten Paar.

\section{Fazit}

Die Durchführung der Unterrichtsstunden nach dem hier vorgeschlagenen Szenario setzt eine fundierte Kenntnis der erwähnten dramapädagogischen Methoden sowie einige Erfahrungen sowohl auf Seiten der Lehrperson als auch bei den Lernenden voraus. Zeitlich einzuplanen ist etwa die doppelte Anzahl von Stunden als bei der herkömmlichen, im Lehrbuch vorgeschlagenen Textarbeit - in meinen Kursen waren dies jeweils 3 Doppelstunden. Im Hinblick auf den Schulalltag ist zu empfehlen, sich auf ein bis drei Techniken zu konzentrieren. Dadurch kann der Unterricht eine interessante Bereicherung erfahren, und die Lernmotivation der Schülerinnen und Schüler kann positiv beeinflusst werden. Voraussetzung für die dramapädagogische Textarbeit ist, dass die Lernenden 
mit Aufwärmübungen auf die szenische Interpretation vorbereitet werden und dass die gemeinsame handlungsorientierte Arbeit abschließend reflektiert wird. Zusammenfassend möchte ich betonen, dass das von mir entwickelte Szenario als ein Vorschlag zu verstehen ist, die herkömmliche Textbucharbeit im Fremdsprachenunterricht zu ergänzen. In Lehrwerken für Deutsch als Fremdsprache findet man zahlreiche Bilder, oft in Verbindung mit Texten. Ob dabei jedoch auch genug Freiräume für eigene Vorstellungen und Deutungen eröffnet werden, wage ich zu bezweifeln. Mein Ziel war es daher aufzuzeigen, dass man auch vom Text ausgehend mit der Erstellung eigener Bilder und damit mit der Bildung von Vorstellungen auf Seiten der Lernenden arbeiten kann, um das Textverständnis nachhaltig zu unterstützen.

\section{Literatur -}

Abraham, Ulf (1999): Vorstellungsbildung und Literaturunterricht. In: Spinner, Kaspar (Hrsg.): Neue Wege im Literaturunterricht. Hannover: Schroedel, 10-20.

Dallapiazza, Rosa-Maria et al. (2002): Tangram 2B. Deutsch als Fremdsprache. Lehrerbuch. Ismaning: Hueber.

Freudenreich, Dorothea; Sperth, Fritz (1983): Stundenblätter. Rollenspiele im Literaturunterricht. Stuttgart: Klett.

Iser, Wolfgang (1984): Der Akt des Lesens. 2. Aufl. München: Fink.

Kunz, Marcel (1995): Szenische Verfahren im Literaturunterricht. In: Spinner, Kaspar (ed.): Imaginative und emotionale Lernprozesse im Deutschunterricht. Frankfurt a.M.: Lang, 55-58.

Madelung, Eva (1996): Vorstellungen als Bausteine unserer Wirklichkeit. Grundlegende Gedanken zum Projekt ,Imaginatives Lernen'. In: Fauser, Peter; Madelung, Eva (eds.): Vorstellungen bilden: Beiträge zum imaginativen Lernen. Seelze-Velber: Kallmeyer, 107-121.

Scheller, Ingo (2004): Szenische Interpretation. Seelze-Velber: Kallmeyer.

Scheller, Ingo (1998): Szenisches Spiel. Handbuch für die pädagogische Praxis. Berlin: Cornelsen.

Scheller, Ingo (1995): Imaginative und emotionale Prozesse bei der szenischen Interpretation von Texten - am Beispiel von Szenen zum Thema ,Jugend und Gewalt‘. In: Spinner, Kaspar (ed.): Imaginative und emotionale Lernprozesse im Deutschunterricht. Frankfurt a.M.: Lang, 59-70.

Schewe, Manfred (1993): Fremdsprache inszenieren. Zur Fundierung einer dramapädagogischen Lehr- und Lernpraxis. Oldenburg: Carl von Ossietzky Universität. 
Spinner, Kaspar (1997): Der Beitrag des Deutschunterrichtes zur Allgemeinbildung. In: Pädagogik 2, 54-57.

Spinner, Kaspar (1993): Von der Notwendigkeit produktiver Verfahren im Literaturunterricht. In: Diskussion Deutsch 134, 491-496. 